\title{
Update in treatment of Symptomatic newly Diagnosed Transplant Eligible Multiple Myeloma Patients
}

\author{
Nahla A M Hamed* \\ Professor of Clinical Hematology, Hematology Department, Faculty of Medicine, Alexandria University, Egypt
}

Submission: December 09, 2019; Published: January 22, 2020

"Correspondence Author: Nahla A M Hamed, Professor of Clinical Hematology, Hematology Department, Faculty of Medicine, Alexandria University, Egypt

\section{Abstract}

\author{
This article tries to answer the following questions \\ i. Is it necessary to incorporate cytogenetic risk categorization in induction decision making? \\ ii. What are the criteria used to assess eligibility for autologous stem-cell transplant (ASCT)? \\ iii. The role of tandem transplantation \\ iv. The role of consolidation and maintenance therapy today \\ v. Is the use of MRD assessment have any role after transplant?
}

Keywords: PET-CT: Positron Emission-Computed Tomography; NDMM: Newly Diagnosed Multiple Myeloma; MDE: Myeloma Defining Events; IMWG: International Myeloma Working Group; ASCT: Autologous Stem-Cell Transplant; FLC: Free Light Chain; CAs: Chromosomal Abnormalities; ISS: International Staging System; LDH: Lactate Dehydrogenase

\section{Introduction}

The updated criteria for multiple myeloma (MM) diagnosis requires evidence of either $10 \%$ or more clonal plasma cells in bone marrow examination or a biopsy-proven plasmacytoma in addition to the presence of one or more myeloma defining events (MDE). MDE consists of established CRAB features (hypercalcemia, renal failure, anemia, or lytic bone lesions) as well as 3 specific biomarkers: clonal bone marrow plasma cells $\geq 60 \%$, serum free light chain (FLC) ratio $\geq 100$ (provided involved FLC level is $\geq 100$ $\mathrm{mg} / \mathrm{L}$ ), and more than one focal lesion on MRI [1].

\section{Important considerations in myeloma diagnosis}

i. The levels of monoclonal (M) proteins and FLC in blood are assessed routinely to confirm active disease [2]. The M protein is considered measurable if it is $\geq 1 \mathrm{~g} / \mathrm{dL}$ in the serum. The serum FLC assay is particularly useful in patients who lack a measurable M protein, provided that the FLC ratio is abnormal and the involved FLC level is $\geq 100 \mathrm{mg} / \mathrm{L}$ [1].

ii. The evaluation of M-protein in urine is less widespread across clinical practice [2]. The M protein is considered measurable if it is $\geq 200 \mathrm{mg} /$ day in the urine [1]. iii. Routine bone marrow assessment (aspirate or biopsy) remains key in the establishment of MM diagnosis in clinical practice, as reflected in the updated International Myeloma Working Group (IMWG) diagnostic criteria [2].

iv. The creatinine clearance test or estimated glomerular filtration rate (calculated using either the Modification of Diet in Renal Disease [MDRD] study or Chronic Kidney Disease Epidemiology Collaboration [CKD-EPI] equations) are also performed at diagnosis to assess renal function [2].

v. Skeletal surveys using $\mathrm{X}$ ray do not reveal lytic bone disease until $70 \%$ of the bone has decalcified [3]. Whole body low dose computed tomography (CT) or magnetic resonance imaging (MRI) is now recommended by the European Myeloma Network (EMN) and the IMWG as standard for the detection of lytic or focal lesions. Both are more sensitive than conventional skeletal survey using $\mathrm{X}$ ray for detecting bone disease [2]. MRI of the spine and pelvis are particularly valuable for detection of focal lesions and may be used if whole body imaging is not available [3]. 
vi. Whole body MRI is also effective for bone marrow evaluation [3].

vii. PET-CTs help in recognizing extramedullary disease, an aggressive phenotype of high-risk myeloma [3].

\section{Risk Status Assessment}

The risk status for all patients should be assessed at the time of diagnosis using the R-ISS. The R-ISS incorporates the original ISS (serum B2M and serum albumin), serum LDH as well as chromosomal abnormalities (CAs) detected by plasma cellspecific interphase FISH. R-ISS stage I is ISS stage I with normal LDH and standard-risk CA. R-ISS stage II is neither stage I nor stage III. R-ISS stage III is stage III ISS (B2M $\geq 5.5 \mathrm{mg} / \mathrm{dL}$ ) with high LDH and/or high-risk CA (del17p, t[4;14], t[14;16]) [4]. In addition to these CAs included in staging, $\mathrm{t}(14 ; 20)$, a gain of $1 \mathrm{q}$ associated with del(1p), and a non hyperdiploid karyotype are considered high-risk features. Greater than or equal to three cytogenetic abnormalities confer ultra-high risk per the IMWG. Gene expression profiling has allowed characterization of gene signatures that also identify high-risk patients with a worse prognosis, but clinical availability of such testing is limited [5].

\section{Treatment of Symptomatic Newly Diagnosed Transplant Eligible Myeloma Patients}

If one or more of MDE are detected in NDMM patients start of therapy is immediately required [6]. Induction regimens have proven efficacious in fit NDMM patients $(<65$ years of age in a good performance status or $<70$ years of age in good clinical condition) [2]. Induction therapy should include the first in class proteasome inhibitor bortezomib combined with dexamethasone (VD) and a third drug preferably an immunomodulatory agent like thalidomide or lenalidomide. Cyclophosphamide or doxorubicin may be an alternative option [6]. The treatment goal should be to induce the deepest possible remission [5]. Typical treatment consists of approximately 3-4 cycles of induction therapy prior to stem cell harvest [1]. After harvest, patients can undergo high-dose chemotherapy with frontline autologous stem cell transplantation as initial consolidation therapy [7]. Alternatively, patients can resume induction therapy delaying ASCT until first relapse [1]. Maintenance therapy should be considered as part of the treatment plan in MM patients due to the known short PFS on no maintenance therapy [4].

\section{Induction Therapy}

\section{What is the preferred induction therapy for patients who are potential candidates for SCT?}

Currently three-drug regimens such as: bortezomib, thalidomide, and dexamethasone (VTd); bortezomib, lenalidomide, and dexamethasone (VRd); bortezomib, cyclophosphamide, and dexamethasone (VCd) [5]; bortezomib, doxorubicin, and dexamethasone (PAD); and carfilzomib, lenalidomide, and dexamethasone (KRd); have all shown improved efficacy compared with two-drug combinations [5]. VCD is a preferred primary treatment option especially in patients with acute renal insufficiency [8]. Cyclophosphamide is an acceptable substitute if an immunomodulatory drug is not immediately available, until it becomes available [4]. In patients presenting with plasma cell leukemia or multiple extramedullary plasmacytomas, initial therapy of VDT-PACE (bortezomib, dexamethasone, thalidomide, cisplatin, doxorubicin, cyclophosphamide, and etoposide) is preferred followed by ASCT and then maintenance with a bortezomib-based regimen [1].

\section{Assessment of treatment Response}

Evaluation of response to therapy is based on the revised IMWG uniform response criteria [1]. All response categories require two consecutive assessments made at any time before starting any new therapy [8]. The M protein level (by SPEP) and serum FLC assay were monitored every month while on therapy [1]. The largest incremental decrease in paraprotein levels is observed following the first cycle of therapy and then, a less steep decline is observed [4]. Urine protein electrophoresis is recommended at least once every 3-6 months, to follow the urine $\mathrm{M}$ protein level as well as to detect other renal complications that may result in albuminuria [1]. Minimal residual disease (MRD) tests should be initiated at the time of suspected complete response based on the revised IMWG uniform response criteria [1]. There is no need for two consecutive assessments for MRD but information on MRD after each treatment stage is required [8]. Positron emission tomography (PET)-CT may be useful in determining treatment response (imaging MRD negative status) and disease progression [2].

\section{What is the optimal number of induction cycles before Transplant?}

The depth of response to induction therapy in NDMM is highly prognostic [4]. Very small incremental decreases in paraprotein are seen beyond three to four cycles of therapy. Therefore, it is recommended that three to four cycles of induction therapy be administered in those planned to proceed to autologous transplant [4].

\section{Mobilization}

Agents associated with stem-cell toxicity should be avoided in patients who are potential candidates for SCT. Melphalan and/ or prolonged immunomodulatory drugs exposure such as [4] lenalidomide beyond four to six cycles may compromise stemcell yield. Some of the deleterious effects from alkylator and lenalidomide exposure can be overcome by either combination of growth factor and chemotherapy or growth factor and CXCR4 antagonist (plerixafor) [4]. Ample stem-cell collection (sufficient for at least 2 transplants) should be considered up front, due to concern for limited ability for future stem-cell collection after prolonged treatment exposure [4]. 


\section{Autologous stem-cell transplant (ASCT)}

ASCT remains integral to the management of NDMM [2]. ASCT increases response rate, depth of response, MRD negativity, and PFS, when used following induction with a novel triplet regimen [6].

\section{What are the criteria used to assess eligibility for autologous stem-cell transplant (ASCT)?}

Eligibility for ASCT is usually based on chronological age, performance state and comorbidities [6]. The level of minimal response required to proceed to SCT is not established for patients receiving induction therapy. Patients should be referred for SCT independent of depth of response [4].

\section{Types of SCT}

SCT may be single autologous SCT (upfront or delayed), a tandem SCT or an allogeneic SCT [8].

\section{Single Autologous SCT}

High dose melphalan / ASCT is performed [4] ideally within 6-8 weeks of finishing induction. High dose melphalan $(200 \mathrm{mg} /$ $\mathrm{m}^{2}$ ) is the standard conditioning regimen for ASCT in multiple myeloma [4]. Melphalan doses may be attenuated for age, frailty, obesity, or renal function. Several studies have used dose-reduced melphalan (70 to $140 \mathrm{mg} / \mathrm{m}^{2}$ ) in older adults [4]. When ASCT is given as part of the planned frontline treatment, $22 \%$ to $44 \%$ patients achieve CR, with median time to progression and OS of 18 to 24 months and 4 to 6 years, respectively with a treatmentrelated mortality of $\sim 2 \%[4]$.

Can transplant be safely delayed until relapse (Salvage or delayed ASCT)?

Delayed SCT may be used as consolidation at first relapse for those not choosing to proceed to transplant initially [4] provided that stem cells are harvested early in the disease course [1]. Several retrospective studies and consensus guidelines suggest that salvage SCT can be a safe and potentially beneficial option, particularly in patients with remission duration of 18 months or more following first ASCT [4]. The IFM 2009 trial reported notable improved PFS and response rate (measured as both CRs and MRD negativity at $1 \times 10^{-4}$ ) in patients who received upfront ASCT compared with those who deferred transplant. Although there were no differences in OS at 4 years, and there were more treatment-related adverse effects in the upfront ASCT arm, there was a substantial population (21\%) of patients in the delayed arm that had refractory disease and are ineligible for transplant at time of relapse [5].

Tandem or double ASCT: a planned second course of high dose therapy and SCT within 6 months of the first course [8]. The role of tandem ASCT remains controversial [6]. Tandem ASCT may be beneficial in patients with high-risk cytogenetic abnormalities [2]; advanced ISS [6] or those with a suboptimal response to first transplant [4]. Those patients who did not achieve a CR or VGPR within 3 months after the first transplant appeared to benefit the most from a second transplant [8].

\section{Allogeneic SCT}

An allogeneic SCT can be performed after prior myeloablative or non myeloablative therapy. Non myeloablative allogeneic transplant (mini transplant) is not adequate and is usually done following maximal tumor control through adequate induction therapy or an autologous SCT. The NCCN guidelines consider myeloablative allogeneic SCT an accepted option, preferably in a clinical trial in:

i) Patients whose disease responds to primary therapy;

ii) Patients with primary PD; or

iii) Patients with PD after an initial autologous SCT [8].

\section{Post-transplant Treatment}

\section{What would be the next recommended step post- transplant?}

\section{Consolidation therapy is recommended now, later, or never?}

Consolidation therapy is not routinely recommended but may be considered in the context of a clinical trial. Consolidation therapy for at least two cycles may be considered for patient's ineligible or unwilling to consider maintenance therapy [4].

\section{What is the role of maintenance therapy?}

Lenalidomide maintenance therapy should be routinely offered to standard-risk patients starting at approximately day 90 to 110 at 10 to $15 \mathrm{mg}$ daily until progression. The optimal duration or depth of response has not been defined. A minimum of 2 years of maintenance therapy irrespective of response is associated with improved PFS. The goal-directed group (stopping lenalidomide once $\mathrm{CR}$ was achieved) received less lenalidomide and was associated with early relapse. Survival benefit has not been clearly shown in patients with ISS stage III disease, those with adverse risk cytogenetics such as $t(4 ; 14)$ or deletion $17 p$, those with elevated LDH, or those with low creatinine clearance [4].

Bortezomib maintenance every 2 weeks may be considered for patients intolerant of or unable to receive lenalidomide. PI with or without lenalidomide may be considered in high-risk patients, especially if bortezomib was part of the initial induction therapy, as this may be associated with improved survival [4].

Evidence is emerging for use of other agents such as ixazomib as maintenance therapy [4].

\section{How can we utilize MRD information in clinical practice?}

Absence of MRD is evidenced ideally by undetectable myeloma cells at levels less than or equal to $1 \times 10^{-6}$ [4]. Skeletal involvement in myeloma has heterogeneous nature. MRD flow only 


\section{Cancer Therapy \& Oncology International Journal}

represents sampling from the pelvic bone, and the disease may be active in other areas of involvement (vertebral bones, skull, long bones, etc.). Patients may be MRD negative by flow cytometry and still positive on PET-CTs. Therefore, PET-CTs are becoming more important [3]. Absence of MRD correlates strongly with PFS and OS [4]. Beyond the prognostic implications of being MRD negative or positive, escalation or de-escalation of therapy based on MRD assay results is not recommended [3]. The only caveat would be an ultrahigh-risk patient who becomes MRD [3].

\section{Is using MRD have any role after transplant?}

Achievement of MRD negativity rates, in the $30-60 \%$ range in ASCT-ineligible and -eligible patients respectively, will hopefully translate in the coming years into furtherly extended PFS and OS and are likely to offer a chance of cure to a fraction of standard risk NDMM [6]. There is insufficient evidence to make modifications to maintenance therapy based on depth of response, including MRD status. Future clinical trials will address whether the MRD status of patients can be used to guide maintenance therapy [4].

\section{Conclusion}

In the ASCT setting, exploration of novel maintenance strategies as well as better platforms are needed to continue to improve outcomes and to give patients with high risk myeloma a better good quality of life, with a minimum burden of therapy.
The role of MRD status in guiding therapy needs to be studied in the future.

\section{References}

1. Rajkumar SV (2018) Multiple myeloma: 2018 update on diagnosis, risk-stratification, and management. Am J Hematol 93(8): 1091-1110.

2. Mateos MV, Ludwig H, Bazarbachi A, Beksac M, Bladé J, et al. (2019) Insights on multiple myeloma treatment strategies. HemaSphere 3: 1 .

3. Usmani SZ, Seifter E (2018) Treatment approach for young, fit, newly diagnosed multiple myeloma patients. Hematology Am Soc Hematol Educ Program 2018(1): 97-102.

4. Mikhael J, Ismaila N, Lacy M, Lipe B, Little RF, et al. (2019) Treatment of multiple myeloma: ASCO and CCO Joint Clinical Practice Guideline. JCO 37(14): 1228-1263.

5. Paul B, Lipe B, Ocio EM, Usmani SZ (2019) Induction therapy for newly diagnosed multiple myeloma. ASCO Educational Book 39: e176-e186.

6. Cavo M, Tacchetti P, Zamagni E, Cellini C, Cangini D, et al. (2019) Frontline treatment of multiple myeloma. Hema Sphere Educational updates in Hematology Book 3(S2): 127-130.

7. Wieduwilt MJ, Walter RB (2019) Clinical hematopoietic cell transplantation and adoptive cell therapy. ASH- SAP American Society of Hematology Self-Assessment Program Textbook. ( $7^{\text {th }}$ edn) pp. 408453.

8. NCCN Clinical Practice Guidelines in Oncology (NCCN Guidelines). Multiple myeloma. Version 1.2020

\section{Your next submission with Juniper Publishers will reach you the below assets}

- Quality Editorial service

- Swift Peer Review

- Reprints availability

- E-prints Service

- Manuscript Podcast for convenient understanding

- Global attainment for your research

- Manuscript accessibility in different formats

( Pdf, E-pub, Full Text, Audio)

- Unceasing customer service

Track the below URL for one-step submission https://juniperpublishers.com/online-submission.php 